PROCEEDINGS OF THE

AMERICAN MATHEMATICAL SOCIETY

Volume 131, Number 2, Pages 359-362

S 0002-9939(02)06599-1

Article electronically published on June 3, 2002

\title{
A SIMPLE PROOF FOR THE FINITENESS OF GIT-QUOTIENTS
}

\author{
ALEXANDER SCHMITT
}

(Communicated by Michael Stillman)

\begin{abstract}
Let $G \times X \longrightarrow X$ be an action of the reductive group $G$ on the projective scheme $X$. For every linearization $\sigma$ of this action in an ample line bundle, there is an open set $X_{\sigma}^{\text {ss }}$ of $\sigma$-semistable points. We provide an elementary and geometric proof for the fact that there exist only finitely many open sets of the form $X_{\sigma}^{\text {ss }}$. This observation was originally due to BiałynickiBirula and Dolgachev and $\mathrm{Hu}$.
\end{abstract}

\section{INTRODUCTION}

Let $\alpha: G \times X \longrightarrow X$ be an action of the reductive group $G$ on the projective scheme $X$ where everything is defined over the algebraically closed field $k$. Geometrically, a linearization of $\alpha$ is a pair $\sigma=(\iota, \rho)$ where $\rho: G \longrightarrow \mathrm{GL}(W)$ is a representation of $G$ on the finite dimensional $k$-vector space $W$ and $\iota: X \hookrightarrow \mathbb{P}(W)$ is an embedding which is equivariant w.r.t. $\alpha$ and the $G$-action on $\mathbb{P}(W)$ coming from $\rho$. To such a linearization, Mumford [4] associates the open subset $X_{\sigma}^{\text {ss }}$ of $\sigma$-semistable points and shows that the universal categorical quotient $\pi: X_{\sigma}^{\mathrm{ss}} \longrightarrow X / / \sigma G$ exists with $X / /{ }_{\sigma} G$ a projective variety. The choice of the linearization is a parameter in the theory. Nevertheless, the following finiteness theorem holds:

Theorem 1.1. Let $\alpha: G \times X \longrightarrow X$ be as above. Then there exist only finitely many open subsets $U \subset X$ for which there is a linearization $\sigma$ of $\alpha$ with

$$
U=X_{\sigma}^{\mathrm{ss}} .
$$

This theorem is due, in characteristic zero, to Białynicki-Birula 1 and Dolgachev and $\mathrm{Hu}$ [3. Białynicki-Birula's paper treats the more general problem of showing that there exist only finitely many " $G$-maximal" open subsets which admit a good quotient in the category of algebraic spaces whereas the approach of Dolgachev and $\mathrm{Hu}$ is based on a very refined study of the Hilbert-Mumford criterion and the structure of one parameter subgroups. We also remark that once one has Theorem 1.1. the analysis of the chamber structure of the $G$-ample cone becomes much easier. Indeed, starting with finitely many linearizations providing the finitely many open subsets $U$ which can occur and applying the master space construction of Thaddeus ([6], cf. also [5]), one is reduced to study a torus action on a projective

Received by the editors April 17, 2001 and, in revised form, September 17, 2001.

1991 Mathematics Subject Classification. Primary 14L24, 14L30.

Key words and phrases. Linearization, semistable points, torus action, Hilbert-Mumford criterion. 
scheme with respect to all possible linearizations in one fixed line bundle (which differ from each other by a character of the torus).

In this note, we shall present an easier proof which should make this important fact more transparent, because it requires only the basic facts of Mumford's Geometric Invariant Theory [4]. In the case $G$ is a torus, the proof is completely elementary and uses only the definition of semistability and the existence of the quotient. Namely, a simple inductive argument reduces everything to the case of $G=\mathbb{G}_{m}$ in which case the semistability sets can be described intrinsically in terms of the fixed point set. This has already been observed by Białynicki-Birula and Sommese [2]. Finally, the general case is derived from the case of a torus by means of the Hilbert-Mumford criterion.

\section{Proof of Theorem 1.1}

Step 1: Reduction to the case of a torus. Fix a maximal torus $T \subset G$ and denote by $\alpha_{T}$ the restriction of $\alpha$ to $T \times X$. Suppose we are given a linearization $\sigma=(\iota, \rho)$ of the action $\alpha$. This provides a linearization $\sigma_{T}=\left(\iota, \rho_{\mid T}\right)$ of $\alpha_{T}$. Our first claim is

$$
X_{\sigma}^{\mathrm{ss}}=\bigcap_{g \in G}\left(g \cdot X_{\sigma_{T}}^{\mathrm{ss}}\right) .
$$

An immediate consequence is

Proposition 2.1. It suffices to prove Theorem 1.1 in the case when $G=T$ is a torus.

To see (1), recall that the Hilbert-Mumford criterion ([4], Thm. 2.1) states that for a closed point $x \in X$

$$
x \in X_{\sigma}^{\text {ss }} \Longleftrightarrow \mu(\lambda, x) \geq 0 \text { for every one parameter subgroup } \lambda: \mathbb{G}_{m} \longrightarrow G \text {. }
$$

Here, $\mu(\lambda, x)$ is defined as the weight with which $\mathbb{G}_{m}$ acts on the fibre of $\mathcal{O}_{\mathbb{P}(W)}(-1)$ over the point $\lim _{z \rightarrow \infty} \lambda(z) \cdot \iota(x)$. Recall that for any $g \in G$, the following equality holds:

$$
\mu\left(g^{-1} \cdot \lambda \cdot g, x\right)=\mu(\lambda, g \cdot x) .
$$

Now, since any two maximal tori are conjugate, every one parameter subgroup of $G$ is conjugate to one of $T$, so that the Hilbert-Mumford criterion can be restated as follows:

$$
\begin{aligned}
x \in X_{\sigma}^{\mathrm{ss}} & \Longleftrightarrow \mu(\lambda, g \cdot x) \geq 0 & \text { for every } \lambda: \mathbb{G}_{m} \longrightarrow T \text { and every } g \in G \\
& \Longleftrightarrow g \cdot x \in X_{\sigma_{T}}^{\mathrm{ss}} & \text { for every } g \in G .
\end{aligned}
$$

This obviously implies (1). The case of a torus $T$ will now be proved by induction over $n=\operatorname{dim} T$.

Step 2: The case $\operatorname{dim} T=1$. First, look at a representation $\rho: \mathbb{G}_{m} \longrightarrow \mathrm{GL}(W)$. The dual representation induces a decomposition $W^{\vee}=W_{1}^{\vee} \oplus \cdots \oplus W_{m}^{\vee}$ where $W_{i}^{\vee}$ is the eigenspace w.r.t. the character $\chi_{d_{i}}: \mathbb{G}_{m} \longrightarrow \mathbb{G}_{m}, z \longmapsto z^{d_{i}}, i=1, \ldots, m$, and we assume $d_{1}<\cdots<d_{m}$. Let $\alpha: \mathbb{G}_{m} \times \mathbb{P}(W) \longrightarrow \mathbb{P}(W)$ be the induced action and 
$\sigma=(\mathrm{i} d, \rho)$ the associated linearization. For $x \in \mathbb{P}(W)$ with lift $w \in W^{\vee}$, we set

$$
\begin{aligned}
d_{\min }(x) & :=\min \left\{d_{i} \mid w \text { has a non-trivial component in } W_{i}^{\vee}\right\}, \\
d_{\max }(x) & :=\max \left\{d_{i} \mid w \text { has a non-trivial component in } W_{i}^{\vee}\right\} .
\end{aligned}
$$

One obviously has

Lemma 2.2. The point $x$ is $\sigma$-semistable if and only if $d_{\min }(x) \leq 0 \leq d_{\max }(x)$.

The set of $\sigma$-semistable points can be described in terms of the paper [2] as follows: Set $F_{i}:=\mathbb{P}\left(W_{i}\right), i=1, \ldots, m$, i.e., $F_{1} \sqcup \cdots \sqcup F_{m}$ is the fixed point locus of the $\mathbb{G}_{m}$-action. Moreover, for $i=1, \ldots, m$, we set

$$
\begin{aligned}
X_{i}^{+} & :=\left\{x \in \mathbb{P}(W) \mid \lim _{z \rightarrow 0} z \cdot x \in F_{i}\right\} \\
& =\mathbb{P}\left(W_{i} \oplus \cdots \oplus W_{m}\right) \backslash \mathbb{P}\left(W_{i+1} \oplus \cdots \oplus W_{m}\right), \\
X_{i}^{-} & :=\left\{x \in \mathbb{P}(W) \mid \lim _{z \rightarrow \infty} z \cdot x \in F_{i}\right\} \\
& =\mathbb{P}\left(W_{1} \oplus \cdots \oplus W_{i}\right) \backslash \mathbb{P}\left(W_{1} \oplus \cdots \oplus W_{i-1}\right) .
\end{aligned}
$$

For every $i<j$, set

$$
\begin{aligned}
C_{i j} & :=\left(X_{i}^{+} \backslash F_{i}\right) \cap\left(X_{j}^{-} \backslash F_{j}\right) \\
& =\mathbb{P}\left(W_{i} \oplus \cdots \oplus W_{j}\right) \backslash\left(\mathbb{P}\left(W_{i+1} \oplus \cdots \oplus W_{j}\right) \cup \mathbb{P}\left(W_{i} \oplus \cdots \oplus W_{j-1}\right)\right) .
\end{aligned}
$$

Rephrasing Lemma 2.2 yields

Corollary 2.3. i) If $d_{i} \neq 0$ for $i=1, \ldots, m$, we set $k:=\max \left\{i \mid d_{i}<0\right\}$. Then

$$
X_{\sigma}^{\mathrm{ss}}=\bigcup_{\substack{1 \leq i \leq k \\ k+1 \leq j \leq m}} C_{i j} .
$$

ii) If, say, $d_{k}=0$, then

$$
X_{\sigma}^{\mathrm{ss}}=X_{k}^{-} \cup X_{k}^{+} \cup \bigcup_{\substack{1 \leq i \leq k-1 \\ k+1 \leq j \leq m}} C_{i j} .
$$

Now, let $\alpha: \mathbb{G}_{m} \times X \longrightarrow X$ be an action of $\mathbb{G}_{m}$ on the projective scheme $X$. Let $F_{1}, \ldots, F_{m}$ be the connected components of the fixed point locus of $\alpha$. Let $\mathcal{P}:=\left(P_{1}, \ldots, P_{s}\right)$ be a tuple of subsets of $\{1, \ldots, m\}$ with $P_{1} \sqcup \cdots \sqcup P_{s}=\{1, \ldots, m\}$. We set $\Gamma_{i}:=\bigcup_{j \in P_{i}} F_{j}, i=1, \ldots, s$. In analogy to the above, we set, for $i=1, \ldots, s$,

$$
\begin{aligned}
& X_{i}^{+}:=\left\{x \in X \mid \lim _{z \rightarrow 0} z \cdot x \in \Gamma_{i}\right\}, \\
& X_{i}^{-}:=\left\{x \in X \mid \lim _{z \rightarrow \infty} z \cdot x \in \Gamma_{i}\right\},
\end{aligned}
$$

and, for $i<j$, we define $C_{i j}:=\left(X_{i}^{+} \backslash \Gamma_{i}\right) \cap\left(X_{j}^{-} \backslash \Gamma_{j}\right)$. Finally, for $k=1, \ldots, s$, we introduce

$$
U(\mathcal{P}, k):=\bigcup_{\substack{1 \leq i \leq k \\ k+1 \leq j \leq s}} C_{i j}
$$

and

$$
U^{0}(\mathcal{P}, k):=X_{k}^{-} \cup X_{k}^{+} \cup \bigcup_{\substack{1 \leq i \leq k-1 \\ k+1 \leq j \leq s}} C_{i j} .
$$


Let $\mathcal{U}$ be the finite collection of subsets of $X$ of the form $U(\mathcal{P}, k)$ or $U^{0}(\mathcal{P}, k)$. Then, restating Corollary 2.3 yields:

Theorem 2.4. Let $\sigma$ be a linearization of the action $\alpha$. Then $X_{\sigma}^{\mathrm{ss}} \in \mathcal{U}$. In particular, Theorem 1.1 holds for $G=\mathbb{G}_{m}$.

Remark 2.5. The above considerations were inspired by the paper [2]. That paper established the finiteness of $\mathbb{G}_{m}$-semistability sets not meeting the fixed point locus. Moreover, studying that paper a little closer, one can narrow the set of partitions $\mathcal{P}$ one has to take into account.

Step 3: Finishing the induction. Let $T$ be an $n$-dimensional torus and write $T=\mathbb{G}_{m} \times T^{\prime}$ where $T^{\prime}$ is a torus of dimension $n-1$. Let $\sigma$ be a linearization of the action $\alpha$ and denote by $\sigma_{1}$ the induced linearization of $\alpha_{\mid \mathbb{G}_{m} \times\{1\}}$. Form the GIT-quotient $\pi: X_{\sigma_{1}}^{\mathrm{ss}} \longrightarrow X / / \sigma_{1} \mathbb{G}_{m}$. By the case $\operatorname{dim} T=1$, there are only finitely many possibilities for $X_{\sigma_{1}}^{\text {ss }}$ and, thus, for $\pi$ and $\bar{X}:=X / / \sigma_{1} \mathbb{G}_{m}$ (up to canonical isomorphy). Now, $\bar{X}$ inherits a $T^{\prime}$-action $\bar{\alpha}$ and a natural linearization $\bar{\sigma}$ of that action. Recall from [5], Prop. 1.3.1, that

$$
X_{\sigma}^{\mathrm{ss}}=X_{\sigma_{1}}^{\mathrm{ss}} \cap \pi^{-1}\left(X_{\bar{\sigma}}^{\mathrm{ss}}\right) .
$$

By induction hypothesis, $X_{\bar{\sigma}}^{\text {ss }}$ belongs to a finite list of open subsets of $\bar{X}$. Since there were only finitely many possibilities for $\bar{X}$ and $\pi$, we are done.

\section{REFERENCES}

[1] A. Białynicki-Birula, Finiteness of the number of maximal open subsets with good quotients, Transform. Groups 3 (1998), 301-319. MR 99m:14089

[2] A. Białynicki-Birula, A.J. Sommese, Quotients by $\mathbb{C}^{*}$ and $\mathrm{SL}(2, \mathbb{C})$ actions, Trans. Amer. Math. Soc. 279 (1983), 773-800. MR 85i:32045

[3] I. Dolgachev, Y. Hu, Variation of geometric invariant theory quotients (With an appendix by Nicolas Ressayre), Inst. Hautes Études Sci. Publ. Math. 87 (1998), 5-56. MR 2000b:14060

[4] D. Mumford, Geometric Invariant Theory, Springer, 1965. MR 35:5451]

[5] Ch. Okonek, A. Schmitt, A. Teleman, Master spaces for stable pairs, Topology 38 (1999), 117-139. MR 99h:14010

[6] M. Thaddeus, Geometric invariant theory and flips, J. Amer. Math. Soc. 9 (1996), 691-723. MR 96m:14017

Universität GH Essen, FB6 Mathematik \& Informatik, D-45117 Essen, Germany 